\title{
EVOLUCIÓN DE LAS TEMPERATURAS Y PRECI- PITACIONES EN LAS CAPITALES DE CASTILLA Y LEÓN EN EL PERÍODO 1961-2006
}

\author{
Antonio CEBALLOS BARBANCHO - Enrique MORÁN TEJEDA - Manuel QUIRÓS \\ HERNÁNDEZ \\ Departamento de Geografía - Universidad de Salamanca
}

Recibido: $24 / 09 / 2007$

Aceptado: $18 / 06 / 2010$

RESUMEN: El presente trabajo analiza la evolución temporal de las principales variables climáticas (temperaturas y precipitaciones) en las 9 capitales provinciales de Castilla y León durante el período 1961-2006. La distribución regular de los observatorios invita a considerar los resultados obtenidos como indicativos de la tendencia del conjunto del territorio regional. Los resultados muestran que las temperaturas medias anuales han experimentado un aumento significativo, especialmente a partir de 1973 , con un incremento promedio de $0,043^{\circ} \mathrm{C}$ por año, debido sobre todo al comportamiento de las temperaturas medias de las máximas. Los meses de marzo y junio han sido los más sensibles a estos cambios. En el caso de las precipitaciones, la mayoría de las series reflejan una tendencia negativa, aunque éstas no sean estadísticamente significativas. Febrero ha sido el mes en el que el descenso de la lluvia ha sido más claro.

$P A L A B R A S C L A V E$ : tendencia temporal, temperaturas, precipitaciones, cambio climático, Castilla y León.

EVOLUTION OF TEMPERATURE AND RAINFALL FROM 1961 TO 2006 IN THE CAPITAL CITIES OF CASTILE AND LEÓN

ABSTRACT: This paper analyses the trend over time of the main climatic variables (temperature and precipitation) in the 9 capital cities of Castilla y León for the period 1961-2006. The homogeneous distribution of the climatic stations throughout the territory has allowed our statistical findings to be representative of data for the whole region. Results showed that the mean annual temperatures have significantly increased, especially since 1973 , with a ratio of $0,043^{\circ} \mathrm{C}$ per year. This was principally due to the behaviour of the maximum temperatures. March and June were the months most sensitive to these changes. In most cases the precipitation series showed a negative trend, although this was not statistically significant. The greatest decrease in precipitation was observed in February.

KEY WORDS: trend over time, temperatures, precipitation, Castilla y León.

ÉVOLUTION DES TEMPÉRATURES ET DES PRÉCIPITATIONS DANS LES CAPITALES DE LA PROVINCE DE CASTILLE-ET-LEON ENTRE 1961-2006

RÉSUMÉ: Le présent travail analyse l'évolution temporaire de celles principales variables climatiques (températures et précipitations) dans les 9 villes capitaux provinciales de Castille et de Leon pendant la période 1961-2006. La distribution régulière des observatoires invite à consi- 
dérer les résultats obtenus comme indicatifs de la tendance de l'ensemble du territoire régional. Les résultats montrent que les températures moyennes annuelles ont éprouvé une augmentation significative, spécialement à partir de 1973 , avec un accroissement moyenne de $0,043^{\circ} \mathrm{C}$ par année, due surtout au comportement des températures maximales moyennes. Les mois de mars et juin ont été ceux plus sensibles à ces changements. Dans le cas des précipitations, bien que la majorité des séries reflètent une tendance négative, toutefois celles-ci ne sont pas statistiquement significatives. Février a été le mois où la diminution de la pluie a été plus claire.

MOTS CLÉ : tendance temporaire, températures, précipitations, changement climatique, Castille et Leon.

\section{EVOLUÇÃO DAS TEMPERATURAS E PRECIPITAÇÕES NAS CAPITAIS DE} CASTELA E LEÃO NO PERIODO 1961 - 2006

RESUMO:. O presente trabalho analisa a evolução temporal das principais variáveis climáticas (temperaturas e precipitações) nas 9 capitais provinciais de Castela e Leão durante o período 1961-2006. A distribuição regular dos observatórios convida a considerar os resultados obtidos como indicativos da tendência do conjunto do território regional. Os resultados mostram que as temperaturas médias anuais experimentaram um aumento significativo, especialmente a partir de 1973, com um incremento médio de $0,043^{\circ} \mathrm{C}$ por ano, devido sobretudo ao comportamento das temperaturas médias das máximas. Os meses de Março e Junho foram os mais sensíveis a estas mudanças. No caso das precipitações, a maioria das séries reflectem uma tendência negativa, embora estas não sejam estatisticamente significativas. Fevereiro foi o mês em que a diminuição da chuva se revelou mais clara.

PALAVRAS CLAVE: tendência temporal, temperaturas, precipitações, mudança climática, Castela e Leão.

\section{INTRODUCCIÓN}

La publicación del avance del último informe del Panel Intergubernamental de Expertos sobre Cambio Climático en febrero de 2007 ha generado una fundada alarma social, ya que éste prevé un aumento medio de temperatura global del planeta de $3^{\circ} \mathrm{C}$ en los próximos años debido al incremento de las concentraciones de gases invernadero. El mismo documento señala que en el caso de las precipitaciones existe una gran heterogeneidad en cuanto a su dinámica espacio-temporal, no obstante los modelos sugieren que entre los $5^{\circ}$ y $45^{\circ}$ de latitud en ambos hemisferios las lluvias van a disminuir en las próximas décadas, mientras que en el resto del planeta experimentarán un cierto incremento, agudizándose el desequilibrio actual en la distribución de la lluvia (IPCC, 2007).

Si por un lado, muchos trabajos de investigación tratan de evaluar el alcance de los cambios futuros mediante la aplicación de modelos, otros están preocupados por describir y analizar los posibles cambios habidos en las últimas décadas para conocer en mayor profundidad el comportamiento del sistema climático y sus consecuencias sobre los sistemas físicos y socio-económicos (p.e. MORENO, 2005). Dentro de esta segunda línea de investigación, destacan trabajos como el de JONES ET AL. (2006) a escala global, el de KLEIN TANK ET AL. 
(2002) a escala continental; los de SERRANO ET AL. (1999), STAUDT (2004), DE CASTRO ET AL. (2005) o BRUNeT ET AL. (2007) para un espacio como la Península Ibérica, y dentro de la propia Península Ibérica estudios regionales como los de Esteban-PARRA ET AL. (1995) en la Meseta Norte, GALÁN ET AL. (2001) en la Meseta Sur, GonZÁLEZ HIDALgO ET AL. (2001) en la Comunidad Valenciana, MORALES ET AL. (2005) en Castilla y León, etc.

Las conclusiones de estos trabajos en muchos casos son diferentes debido a las distintas escalas de análisis, las características técnicas de las series de datos utilizadas (duración del período de análisis, número de observatorios, método de homogenización), las técnicas de análisis estadístico empleadas e incluso la propia variabilidad espacio-temporal de temperaturas y, especialmente, de precipitaciones.

El objetivo general del presente trabajo es analizar la evolución temporal, y el significado estadístico de la misma, de las temperaturas medias, temperaturas medias de las máximas, temperaturas medias de las mínimas y precipitaciones, tanto mensuales como anuales, correspondientes a los nueve observatorios de las capitales provinciales de Castilla y León. Aunque el número de observatorios meteorológicos utilizado en el análisis es limitado, la distribución regular de los mismos en el territorio castellano-leonés invita a considerar los resultados obtenidos como indicativos de la tendencia general para el conjunto de Castilla y León (FIGURA 1). Además, los datos disponibles permiten la generación de series homogeneizadas para el período 1961-2006 $(\mathrm{n}=46)$, incluyendo por tanto en el análisis los datos correspondientes a los últimos 10-15 años, precisamente aquellos que a nivel mundial han registrado las mayores anomalías respecto a las medias del período climático normal 1961-90 y que no han sido tratados en la mayor parte de los trabajos publicados. Finalmente, se discuten los resultados obtenidos en relación a los publicados por otros autores en un contexto espacial próximo.

\section{MÉTODOS Y TÉCNICAS}

Las series de datos termo-pluviométricos correspondientes a las nueve capitales de Castilla y León (CUADRO 1) han sido homogeneizadas de acuerdo con los siguientes criterios:

i) Selección de un período homogéneo común a todos de al menos 45 años (1961-2006), incluyendo el último período climático normal (1961-90) y los años más recientes (últimos años de los noventa y primeros años del siglo XXI) debido a que no suelen estar incluidos en los trabajos de análisis regional publicados en los últimos años y a que son los años más anómalos en el conjunto de registros. 
ii) Identificación de outliers siguiendo la propuesta de AGUILAR ET AL. (1999) y SALADIÉ ET AL. (2002), considerando como outliers aquellos valores, bien mensuales o anuales, situados por encima o por debajo de $4 \sigma$ de la media. Los valores considerados outliers se compararon con los del resto de estaciones y si en algunas de estas también había datos fuera de rango, todos eran considerados como correctos.

iii) Reconstrucción de lagunas. A pesar de que el porcentaje de meses sin datos en ningún caso ha superado el $2 \%$ respecto al volumen total de datos, los datos ausentes han sido estimados a partir de correlaciones lineales con observatorios vecinos con un nivel de confianza superior al 95 \% (TOMÁs ET AL., 1998).

iv) Las series temporales de datos climáticos han sido correlacionadas entre sí mediante la aplicación del coeficiente de correlación Pearson, con un intervalo de confianza superior al $95 \%$, ya que la aplicación de este test a modo de filtro permite evaluar el grado de consistencia espacial de los resultados obtenidos (SHORTHOUSE Y ARNELL, 1999).

Cuadro 1. Localización de los observatorios meteorológicos, pertenecientes a la Agencia Estatal de Meteorología (AEMET), utilizados en este estudio.

\begin{tabular}{lllllc}
\hline Capital & Observatorio & ID & Latitud & Longitud & Altitud $(\mathrm{m})$ \\
\hline Ávila & idem & 2444 & $40^{\circ} 39^{\prime} 00^{\prime \prime} \mathrm{N}$ & $04^{\circ} 42^{\prime} 00^{\prime \prime} \mathrm{W}$ & 1132 \\
Burgos & Villafría & 2331 & $42^{\circ} 21^{\prime} 22^{\prime \prime} \mathrm{N}$ & $03^{\circ} 37^{\prime} 57^{\prime \prime} \mathrm{W}$ & 890 \\
León & Virgen del Camino & 2661 & $42^{\circ} 35^{\prime} 20^{\prime \prime} \mathrm{N}$ & $05^{\circ} 38^{\prime} 58^{\prime \prime} \mathrm{W}$ & 916 \\
Palencia & Escuela de Cap. Agraria & 2401 & $42^{\circ} 00^{\prime} 30^{\prime \prime} \mathrm{N}$ & $04^{\circ} 33^{\prime} 27^{\prime \prime} \mathrm{W}$ & 760 \\
Palencia & Monzón de Campos & 2386 & $42^{\circ} 07^{\prime} 00^{\prime \prime} \mathrm{N}$ & $04^{\circ} 29^{\prime} 37^{\prime \prime} \mathrm{W}$ & 754 \\
Salamanca & Matacán & 2867 & $40^{\circ} 46^{\prime} 50^{\prime \prime} \mathrm{N}$ & $05^{\circ} 29^{\prime} 50^{\prime} \mathrm{W}$ & 790 \\
Segovia & idem & 2465 & $40^{\circ} 56^{\prime} 52^{\prime \prime} \mathrm{N}$ & $04^{\circ} 07^{\prime} 38^{\prime \prime} \mathrm{W}$ & 1005 \\
Soria & idem & 2030 & $41^{\circ} 46^{\prime} 00^{\prime \prime} \mathrm{N}$ & $02^{\circ} 28^{\prime} 00^{\prime \prime} \mathrm{W}$ & 1082 \\
Valladolid & Villanubla & 2539 & $41^{\circ} 42^{\prime} 00^{\prime \prime} \mathrm{N}$ & $04^{\circ} 51^{\prime} 00^{\prime \prime} \mathrm{W}$ & 846 \\
Zamora & idem & 2614 & $41^{\circ} 31^{\prime} 00^{\prime \prime} \mathrm{N}$ & $05^{\circ} 44^{\prime} 01^{\prime \prime} \mathrm{W}$ & 656 \\
\hline
\end{tabular}

Una vez homogeneizadas las series de datos se procedió al siguiente análisis:

i) Para determinar las anomalías térmicas se calculó la diferencia en ${ }^{\circ} \mathrm{C}$ entre la temperatura media diaria, de las máximas y de las mínimas, correspondiente a cada año y mes, y la media respectiva del último periodo climático normal (CLINO 1961-90). El resultado se ha expresado gráficamente observando la tendencia mediante la representación de la media móvil de 5 años (IPCC, 2001). $\mathrm{Pa}-\mathrm{a}$ constatar el grado de significación de la tendencia (positiva, negativa o nula), con un determinado nivel de confianza, se aplicó un test no parámetrico como la correlación de rangos de Spearman-Rs-(SNEYERS, 1992; YUE ET AL., 2002).

ii) Una vez comprobada la distribución normal de la mayoría de las series de precipitaciones, para estudiar la tendencia de las mismas, en primer lugar, se de- 
terminó la dispersión de la muestra observando la frecuencia de años secos y húmedos según proponen SCIAN Y DONNARI (1997), para quienes año seco es aquel con valores inferiores a la precipitación media anual menos la desviación estándar y año húmedo es aquel con un registro superior a la media más la desviación estándar. En segundo lugar, para examinar la tendencia temporal de las series anuales y mensuales, así como su grado de significación, como en el caso de las temperaturas, se aplicó el test de Spearman, cuantificándose, además, el alcance del cambio mediante un ajuste lineal.

\section{RESULTADOS}

\section{Homogenización de la serie y breve caracterización climática}

Una vez completas todas las series de datos, de acuerdo al protocolo expuesto en el apartado previo, éstas han sido correlacionadas entre sí para tener una aproximación del significado espacial de las tendencias observadas.

En el caso de las temperaturas medias de las máximas (CUADRO 2a) existe una muy buena correlación entre todas las series (con un nivel de confianza superior al $99 \%$ ) con la excepción de Palencia, que en varios casos mantiene una relación nula con el resto de observatorios. Las correlaciones entre las series correspondientes a las temperaturas medias de las mínimas (CUADRO 3a) reflejan, en general, unos coeficientes de correlación notablemente inferiores que los incluidos en el CUADRO 2a. Aunque Palencia sigue mostrando los coeficientes de correlación más bajos, en este caso el observatorio de Ávila también apunta un comportamiento diferente respecto al resto. Con toda probabilidad ello se deba a las siguientes circunstancias: i) Ávila es el observatorio situado a mayor altitud de todos los estudiados (CUADRO 1); ii) en el caso de la cuenca del Duero, las temperaturas mínimas, especialmente entre los meses de noviembre y marzo, dependen de un meteoro de carácter local como las nieblas, con una incidencia desigual en las distintas zonas dependiendo de la topografía y la altitud. El análisis de correlación entre las series correspondientes a las temperaturas medias muestra un nivel de correlación significativo en todos los casos, con un nivel de confianza superior al $99 \%$ salvo Palencia (CUADRO 4a). Revisando la serie histórica de Palencia capital hemos comprobado que el observatorio ha tenido tres ubicaciones distintas en las últimas cuatro décadas. Aunque hemos analizado el nivel de correlación entre las tres series, en aquellos intervalos de tiempo en que se midieron las temperaturas simultáneamente, y corregido el sesgo inducido por el cambio de localización, el resultado de los CUADROS 2a, 3a y 4a no satisface los criterios de calidad expuestos en el apartado 2 por lo que hemos buscado una serie alternativa que sea comparable a Palencia capital y cumpla con los criterios de calidad exigidos. Por este motivo en el análisis de las temperaturas hemos utilizado la serie correspondiente a la estación de la lo- 
calidad de Monzón de Campos, situada a $10 \mathrm{~km}$ al norte de la ciudad de Palencia y con una situación muy parecida al observatorio de la capital (CUADRO 1). Sustituyendo en los análisis de correlación lineal los datos de Palencia por los de Monzón de Campos los coeficientes de correlación de Pearson aumentan de manera ostensible y siempre significativos al $99 \%$ (CUADROS $2 b, 3$ y y $4 b$ ).

Cuadro 2. Análisis de correlación simple entre las distintas series de datos anuales de temperaturas medias de las máximas.

\begin{tabular}{|c|c|c|c|c|c|c|c|c|c|c|c|c|c|c|c|c|c|c|c|}
\hline & \multicolumn{9}{|c|}{ Cuadro a: Temperaturas medias de las máximas } & \multicolumn{10}{|c|}{ Cuadro b: Temperaturas medias de las máximas } \\
\hline & $\gtreqless$ & $\stackrel{D}{ }$ & 犲 & $\lesssim$ & $\underset{\sim}{\ll}$ & ע & $\overbrace{n}$ & $\$$ & 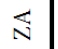 & & $\gtreqless$ & $\stackrel{D}{\sim}$ & 桴 & $\lesssim$ & $\ll$ & ש্র & O & $\lesssim$ & $\$$ \\
\hline AV & & 0,88 & 0,71 & 0,07 & 0,92 & 0,96 & 0,89 & 0,87 & 0,90 & $\mathrm{AV}$ & & 0,88 & 0,71 & 0,82 & 0,92 & 0,96 & 0,89 & 0,87 & 0,90 \\
\hline $\mathrm{BU}$ & & & 0,67 & 0,29 & 0,86 & 0,91 & 0,88 & 0,85 & 0,90 & BU & & & 0,67 & 0,82 & 0,86 & 0,91 & 0,88 & 0,85 & 0,90 \\
\hline LE & & & & 0,29 & 0,81 & 0,80 & 0,84 & 0,89 & 0,75 & LE & & & & 0,86 & 0,81 & 0,80 & 0,84 & 0,89 & 0,75 \\
\hline PA & & & & & 0,08 & 0,17 & 0,20 & 0,30 & 0,22 & $\mathrm{PA}$ & & & & & 0,88 & 0,90 & 0,87 & 0,96 & 0,86 \\
\hline SA & & & & & & 0,95 & 0,88 & 0,92 & 0,93 & SA & & & & & & 0,95 & 0,88 & 0,92 & 0,93 \\
\hline SG & & & & & & & 0,91 & 0,93 & 0,92 & SG & & & & & & & 0,91 & 0,93 & 0,92 \\
\hline SO & & & & & & & & 0,92 & 0,84 & SO & & & & & & & & 0,92 & 0,84 \\
\hline VA & & & & & & & & & 0,91 & VA & & & & & & & & & 0,91 \\
\hline ZA & & & & & & & & & & $\mathrm{ZA}$ & & & & & & & & & \\
\hline
\end{tabular}

Cuadro 3. Análisis de correlación simple entre las distintas series de datos anuales de temperaturas medias de las mínimas.

\begin{tabular}{|c|c|c|c|c|c|c|c|c|c|c|c|c|c|c|c|c|c|c|c|}
\hline & \multicolumn{9}{|c|}{ Cuadro a: Temperaturas medias de las mínimas } & \multicolumn{10}{|c|}{ Cuadro b: Temperaturas medias de las mínimas } \\
\hline & $\gtreqless$ & $\stackrel{p}{m}$ & 됙 & 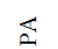 & 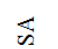 & $\underset{\mathscr{N}}{0}$ & O & $\lesseqgtr$ & 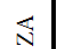 & & $\gtreqless$ & $\vec{m}$ & 됙 & $\lesssim$ & $\ll$ & $\underset{\mathscr{N}}{0}$ & 0 & $\lesseqgtr$ & 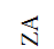 \\
\hline AV & & 0,49 & 0,04 & 0,39 & 0,13 & 0,58 & 0,29 & 0,41 & 0,13 & AV & & 0,49 & 0,04 & 0,44 & 0,13 & 0,58 & 0,29 & 0,41 & 0,13 \\
\hline BU & & & 0,72 & 0,50 & 0,72 & 0,74 & 0,88 & 0,86 & 0,80 & BU & & & 0,72 & 0,83 & 0,72 & 0,74 & 0,88 & 0,86 & 0,80 \\
\hline LE & & & & 0,14 & 0,77 & 0,66 & 0,88 & 0,82 & 0,86 & LE & & & & 0,46 & 0,77 & 0,66 & 0,88 & 0,82 & 0,86 \\
\hline PA & & & & & 0,21 & 0,33 & 0,39 & 0,39 & 0,25 & $\mathrm{PA}$ & & & & & 0,61 & 0,65 & 0,62 & 0,65 & 0,69 \\
\hline SA & & & & & & 0,69 & 0,68 & 0,85 & 0,92 & SA & & & & & & 0,69 & 0,68 & 0,85 & 0,92 \\
\hline SG & & & & & & & 0,72 & 0,81 & 0,70 & SG & & & & & & & 0,72 & 0,81 & 0,70 \\
\hline SO & & & & & & & & 0,84 & 0,80 & SO & & & & & & & & 0,84 & 0,80 \\
\hline VA & & & & & & & & & 0,87 & VA & & & & & & & & & 0,87 \\
\hline ZA & & & & & & & & & & ZA & & & & & & & & & \\
\hline
\end{tabular}

Cuadro a: análisis con los datos correspondientes a la series de los observatorios de Palencia capital. Cuadro b: Análisis sustituyendo los datos de Palencia capital por los de Monzón de Campos

Blanco: correlación no significativa. Gris claro: tendencia significativa con un nivel de confianza del 95

$\%$. Gris oscuro: tendencia significativa con un nivel de confianza del $99 \%$ 
Los coeficientes de correlación correspondientes a las series pluviométricas son significativos en casi todos los casos con un nivel de confianza del $99 \%$. Sólo en dos ocasiones el nivel confianza bajó de este umbral, aunque por encima del $95 \%$ (CUADRO 5).

Los valores medios de temperaturas y precipitaciones para el período 19612006 (46 años) correspondientes a los 9 observatorios estudiados aparecen en el CUADRO 6. Las temperaturas medias anuales oscilan entre 10,3 y $12,8^{\circ} \mathrm{C}$, siempre en el intervalo del bioclima supramediterráneo representativo de la mayor parte de la comunidad castellano-leonesa. Enero es el mes más frío, con temperaturas medias en torno a $\operatorname{los} 3,5^{\circ} \mathrm{C}$, mientras que el mes más cálido es julio con una temperatura media de unos $20,5^{\circ} \mathrm{C}$. La existencia de inviernos fríos, veranos relativamente frescos, una oscilación térmica anual superior a los $17^{\circ}$ $\mathrm{C}$, una precipitación anual por debajo de la isoyeta de $\operatorname{los} 600 \mathrm{~mm}$ en gran parte del territorio, y el registro de al menos 2 meses secos al año (consideramos mes seco cuando el valor correspondiente al doble de su temperatura media expresada en ${ }^{\circ} \mathrm{C}$ supera al valor de la precipitación total expresado en $\mathrm{mm}$ ), permiten clasificar al clima de nuestro territorio como mediterráneo seco con un claro matiz continental inducido por el efecto combinado de la altitud, la longitud y el aislamiento orográfico.

Cuadro 4. Análisis de correlación simple entre las distintas series de datos anuales de temperaturas medias. Cuadro a: análisis con los datos correspondientes a la series de los observatorios de Palencia capital. Cuadro b: Análisis sustituyendo los datos de Palencia capital por los de Monzón de Campos

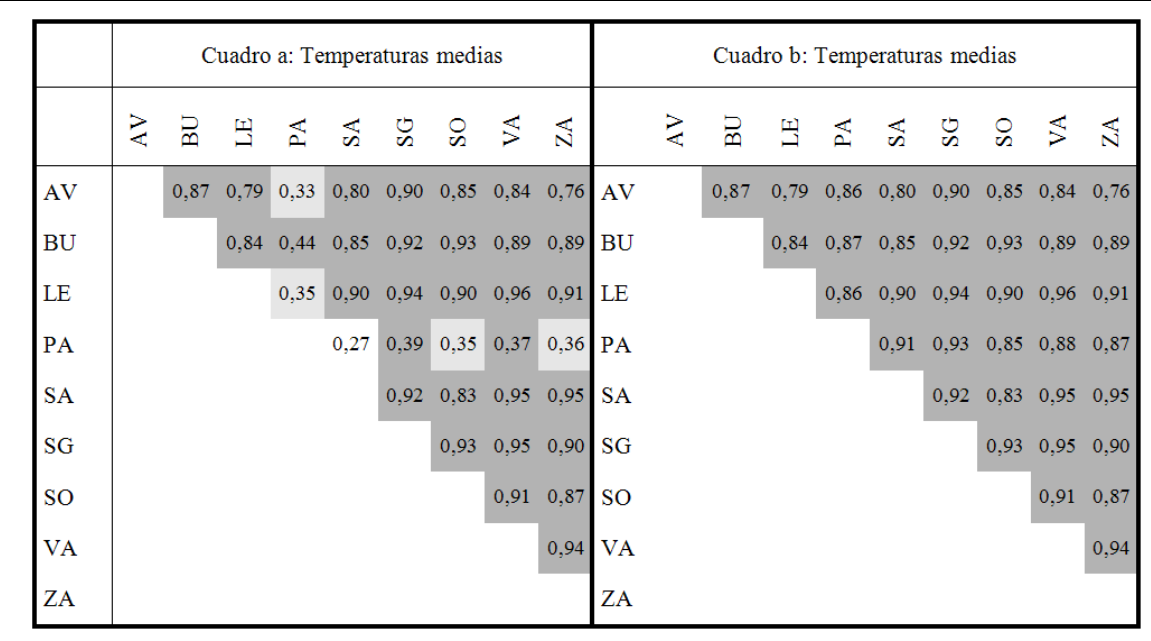

Blanco: correlación no significativa. Gris claro: tendencia significativa con un nivel de confianza del 95 $\%$. Gris oscuro: tendencia significativa con un nivel de confianza del $99 \%$ 
Cuadro 5. Análisis de correlación simple entre las distintas series de datos anuales de precipitaciones.

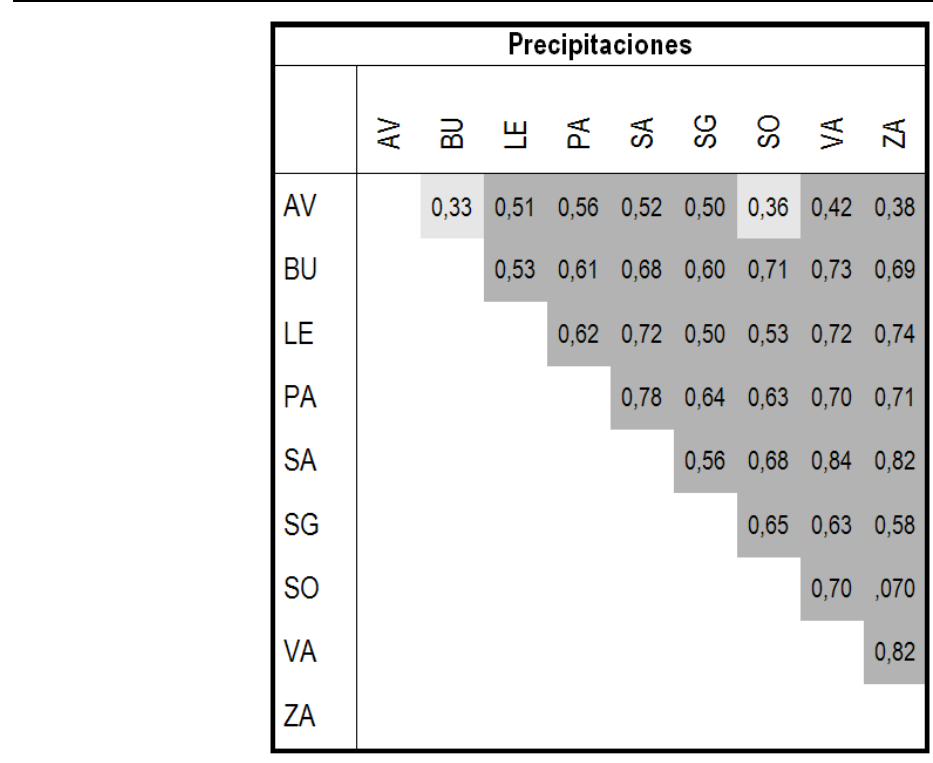

Blanco: correlación no significativa. Gris claro: tendencia significativa con un nivel de confianza del 95 $\%$. Gris oscuro: tendencia significativa con un nivel de confianza del $99 \%$

Cuadro 6. Valores medios $\left({ }^{\circ} \mathrm{C}\right)$, coeficientes de variación (\%) de temperaturas y precipitaciones correspondientes a las 9 capitales de provincia castellano-leonesas durante el periodo 1961-2006.

\begin{tabular}{lcccccccc}
\hline \multicolumn{1}{c}{ Capital } & \multicolumn{2}{c}{ T. media } & \multicolumn{2}{c}{ T. media máx. } & \multicolumn{2}{c}{ T. media mín. } & \multicolumn{2}{c}{ Precipitación } \\
\hline Ávila & Media & $C V$ & Media & $C V$ & Media & $C V$ & Media) & $C V$ \\
Burgos & 10,6 & 7,5 & 16,0 & 7,5 & 5,2 & 17,3 & 380,4 & 26,5 \\
León & 10,3 & 7,8 & 16,0 & 6,9 & 4,5 & 13,3 & 563,6 & 16,6 \\
Palencia* & 10,9 & 5,5 & 16,6 & 3,6 & 5,2 & 11,5 & 544,0 & 21,9 \\
Salamanca & 11,2 & 7,1 & 17,4 & 4,6 & 5,0 & 16,0 & 430,2 & 24,0 \\
Segovia & 11,8 & 5,9 & 18,1 & 4,4 & 5,5 & 10,9 & 380,3 & 21,7 \\
Soria & 11,8 & 5,9 & 17,2 & 5,2 & 6,7 & 7,5 & 456,6 & 23,2 \\
Valladolid & 10,7 & 6,5 & 16,8 & 4,8 & 4,6 & 13,0 & 516,0 & 20,0 \\
Zamora & 11,2 & 5,4 & 17,1 & 4,7 & 4,9 & 12,2 & 456,6 & 24,0 \\
\hline
\end{tabular}

* Datos correspondientes a la localidad de Monzón de Campos (Palencia)

\section{Comportamiento de las temperaturas}

Las temperaturas medias anuales durante la totalidad del período de observación han mostrado una tendencia positiva en todos los observatorios, con un nivel de confianza del 99\% (CUADRO 7a). La FIGURA 2 refleja la evolución temporal de las anomalías térmicas en las nueve capitales de provincia, siendo la trayectoria de la línea correspondiente a las temperaturas medias anuales muy 
parecida, con un punto de inflexión claro en los años 1972 y 1973 . Entre 1961 y 1972 las temperaturas medias experimentan un descenso y a partir de ese momento, y hasta el año 2006, la tendencia es claramente positiva. Si en el análisis estadístico sólo consideramos el período 1973-2006 ( $\mathrm{n}=34)$ el valor de los coeficientes de correlación de Spearman aumenta de forma clara (CUADRO 7b) y un ajuste lineal permite cuantificar el alcance real de este incremento que oscilaría entre los $0,065^{\circ} \mathrm{C} /$ año de Burgos y los 0,024 de León, con un promedio para el conjunto de los 9 observatorios de $0,043^{\circ} \mathrm{C} / \mathrm{año}$. Analizando los años más cálidos de cada una de las localidades, podemos concluir que 2006, 1997, 2003 y 1995 han sido los que han registrado los valores más altos para el conjunto de la serie, coincidiendo con la apreciación general del IPCC (2007) que destaca que en las dos últimas décadas se han medido los valores de temperaturas más altos en gran parte de las estaciones de la red mundial.

Cuadro 7. Coeficientes de correlación de Spearman correspondientes a las series de temperaturas medias mensuales y anuales. Cuadro a: resultados correspondientes a la totalidad del periodo de estudio (1961-2006). Cuadro b: resultados correspondientes al periodo 1973-2006.

\begin{tabular}{|c|c|c|c|c|c|c|c|c|c|c|c|c|c|}
\hline \multicolumn{14}{|c|}{ Cuadro a: Temperatura medias (1961-2006) } \\
\hline & Ene & $\mathrm{Feb}$ & Mar & $A b r$ & May & Jun & Jul & Ago & Sep & Oct & Nov & Dic & Año \\
\hline Ávila & $-0,13$ & 0,01 & 0,44 & 0,25 & 0,20 & 0,44 & 0,23 & 0,27 & $-0,02$ & 0,07 & 0,14 & 0,24 & 0,38 \\
\hline Burgos & 0,24 & 0,09 & 0,56 & 0,34 & 0,43 & 0,57 & 0,47 & 0,55 & 0,15 & 0,20 & 0,24 & 0,33 & 0,67 \\
\hline León & 0,07 & 0,07 & 0,44 & 0,12 & 0,16 & 0,40 & 0,10 & 0,17 & $-0,05$ & $-0,04$ & 0,15 & 0,24 & 0,45 \\
\hline Palencia & 0,12 & 0,04 & 0,38 & 0,05 & 0,13 & 0,38 & 0,09 & 0,23 & $-0,04$ & 0,05 & 0,10 & 0,33 & 0,39 \\
\hline Salamanca & 0,03 & 0,06 & 0,45 & 0,11 & 0,20 & 0,45 & 0,18 & 0,27 & 0,01 & 0,11 & 0,22 & 0,27 & 0,43 \\
\hline Segovia & 0,06 & 0,11 & 0,44 & 0,14 & 0,10 & 0,44 & 0,01 & 0,21 & 0,03 & 0,14 & 0,13 & 0,35 & 0,49 \\
\hline Soria & 0,16 & 0,11 & 0,52 & 0,21 & 0,26 & 0,47 & 0,24 & 0,33 & $-0,01$ & 0,11 & 0,26 & 0,33 & 0,57 \\
\hline Valladolid & 0,04 & 0,03 & 0,45 & 0,14 & 0,20 & 0,45 & 0,26 & 0,26 & 0,00 & $-0,01$ & 0,11 & 0,24 & 0,49 \\
\hline Zamora & 0,06 & 0,00 & 0,52 & 0,28 & 0,34 & 0,54 & 0,38 & 0,41 & 0,08 & 0,10 & 0,21 & 0,27 & 0,62 \\
\hline \multicolumn{14}{|c|}{ Cuadro b: Temperatura medias (1973-2006) } \\
\hline & Ene & $\mathrm{Feb}$ & Mar & $A b r$ & May & Jun & Jul & Ago & Sep & Oct & Nov & Dic & Año \\
\hline Ávila & $-0,02$ & 0,05 & 0,56 & 0,58 & 0,59 & 0,65 & 0,60 & 0,51 & 0,01 & 0,31 & 0,08 & $-0,03$ & 0,77 \\
\hline Burgos & 0,15 & 0,04 & 0,58 & 0,56 & 0,63 & 0,70 & 0,55 & 0,48 & 0,26 & 0,47 & 0,25 & 0,08 & 0,81 \\
\hline León & $-0,12$ & $-0,03$ & 0,44 & 0,26 & 0,41 & 0,52 & 0,21 & 0,12 & $-0,08$ & 0,21 & $-0,02$ & $-0,02$ & 0,48 \\
\hline Palencia & 0,13 & $-0,02$ & 0,47 & 0,41 & 0,44 & 0,53 & 0,27 & 0,28 & 0,11 & 0,38 & 0,16 & 0,14 & 0,68 \\
\hline Salamanca & 0,07 & $-0,03$ & 0,55 & 0,40 & 0,48 & 0,63 & 0,44 & 0,34 & 0,11 & 0,41 & 0,25 & 0,08 & 0,69 \\
\hline Segovia & $-0,03$ & 0,08 & 0,48 & 0,37 & 0,37 & 0,60 & 0,31 & 0,29 & 0,05 & 0,33 & 0,00 & 0,05 & 0,69 \\
\hline Soria & 0,04 & 0,08 & 0,52 & 0,44 & 0,50 & 0,59 & 0,32 & 0,26 & 0,01 & 0,34 & 0,12 & 0,06 & 0,69 \\
\hline Valladolid & 0,00 & $-0,07$ & 0,49 & 0,34 & 0,47 & 0,60 & 0,37 & 0,20 & $-0,01$ & 0,24 & 0,01 & $-0,02$ & 0,62 \\
\hline Zamora & 0,07 & $-0,15$ & 0,51 & 0,44 & 0,58 & 0,66 & 0,46 & 0,30 & 0,03 & 0,35 & 0,15 & 0,08 & 0,69 \\
\hline
\end{tabular}

Blanco: correlación no significativa. Gris claro: tendencia significativa con un nivel de confianza del 95 $\%$. Gris oscuro: tendencia significativa con un nivel de confianza del $99 \%$

El análisis intraanual de las temperaturas medias muestra de manera inequívoca que los meses más sensibles al aumento de las temperaturas han sido marzo y junio (CUADRO 7a), aunque si nos atenemos a lo ocurrido en las últimas tres décadas (CUADRO 7b) el aumento significativo de las temperaturas es generalizable al conjunto de los meses primaverales y la primera parte del verano. Para el conjunto del período (1961-2006) las temperaturas subieron en marzo y 
junio 0,053 y $0,060^{\circ} \mathrm{C} /$ año respectivamente. Considerando el subperíodo $1973-$ 2006, el ritmo de la subida es mayor: $0,077^{\circ} \mathrm{C} /$ año en marzo y $0,107^{\circ} \mathrm{C} /$ año en junio. En el caso de los meses invernales no se ha observado ninguna tendencia estadísticamente significativa, salvo en el mes de diciembre en donde sólo en la serie completa y en 4 de los 9 observatorios la tendencia ha sido positiva con un intervalo de confianza del $95 \%$.

Cuadro 8. Coeficientes de correlación de Spearman correspondientes a las series de temperaturas medias máximas mensuales y anuales. Cuadro a: resultados correspondientes a la totalidad del periodo de estudio (1961-2006). Cuadro b: resultados correspondientes al periodo 1973-2006

\begin{tabular}{|c|c|c|c|c|c|c|c|c|c|c|c|c|c|}
\hline \multicolumn{14}{|c|}{ Cuadro a: Temperatura medias de las máximas (1961-2006) } \\
\hline & Ene & $\mathrm{Feb}$ & Mar & $A b r$ & May & Jun & Jul & Ago & Sep & Oct & Nov & Dic & Año \\
\hline Ávila & 0,15 & 0,28 & 0,52 & 0,36 & 0,23 & 0,64 & 0,49 & 0,51 & 0,22 & 0,25 & 0,37 & 0,40 & 0,67 \\
\hline Burgos & 0,38 & 0,26 & 0,59 & 0,41 & 0,43 & 0,59 & 0,52 & 0,56 & 0,17 & 0,15 & 0,21 & 0,49 & 0,75 \\
\hline León & 0,00 & 0,18 & 0,36 & 0,06 & 0,05 & 0,30 & $-0,10$ & $-0,05$ & $-0,09$ & $-0,18$ & 0,04 & 0,17 & 0,12 \\
\hline Palencia & 0,11 & 0,13 & 0,46 & 0,17 & 0,17 & 0,47 & 0,20 & 0,13 & $-0,04$ & $-0,10$ & 0,08 & 0,30 & 0,41 \\
\hline Salamanca & 0,20 & 0,23 & 0,48 & 0,12 & 0,07 & 0,45 & 0,14 & 0,20 & 0,05 & 0,06 & 0,25 & 0,49 & 0,53 \\
\hline Segovia & 0,27 & 0,24 & 0,47 & 0,22 & 0,06 & 0,49 & 0,16 & 0,28 & 0,10 & 0,17 & 0,29 & 0,49 & 0,60 \\
\hline Soria & 0,17 & 0,23 & 0,46 & 0,17 & 0,16 & 0,43 & 0,22 & 0,22 & $-0,02$ & $-0,02$ & 0,23 & 0,44 & 0,49 \\
\hline Valladolid & 0,00 & 0,13 & 0,45 & 0,19 & 0,15 & 0,50 & 0,25 & 0,18 & 0,00 & $-0,09$ & 0,09 & 0,30 & 0,46 \\
\hline Zamora & 0,11 & 0,19 & 0,55 & 0,35 & 0,27 & 0,56 & 0,42 & 0,40 & 0,13 & 0,05 & 0,14 & 0,39 & 0,68 \\
\hline \multicolumn{14}{|c|}{ Cuadro b: Temperatura medias de las máximas (1973-2006) } \\
\hline & Ene & Feb & Mar & $A b r$ & May & Jun & Jul & Ago & Sep & Oct & Nov & Dic & Año \\
\hline Ávila & 0,13 & 0,34 & 0,56 & 0,57 & 0,54 & 0,78 & 0,58 & 0,55 & 0,24 & 0,43 & 0,19 & 0,16 & 0,82 \\
\hline Burgos & 0,19 & 0,20 & 0,57 & 0,54 & 0,65 & 0,69 & 0,55 & 0,45 & 0,17 & 0,32 & 0,00 & 0,12 & 0,80 \\
\hline León & $-0,18$ & 0,20 & 0,31 & 0,16 & 0,34 & 0,48 & 0,10 & $-0,03$ & $-0,13$ & 0,09 & $-0,12$ & $-0,04$ & 0,38 \\
\hline Palencia & $-0,05$ & 0,06 & 0,36 & 0,33 & 0,43 & 0,51 & 0,17 & 0,04 & $-0,10$ & 0,05 & $-0,08$ & $-0,02$ & 0,48 \\
\hline Salamanca & 0,28 & 0,27 & 0,44 & 0,28 & 0,39 & 0,65 & 0,39 & 0,33 & 0,14 & 0,28 & 0,14 & 0,29 & 0,78 \\
\hline Segovia & 0,14 & 0,24 & 0,47 & 0,40 & 0,37 & 0,66 & 0,38 & 0,38 & 0,13 & 0,32 & 0,05 & 0,17 & 0,75 \\
\hline Soria & 0,13 & 0,27 & 0,46 & 0,30 & 0,44 & 0,56 & 0,31 & 0,19 & $-0,03$ & 0,13 & $-0,04$ & 0,23 & 0,67 \\
\hline Valladolid & $-0,12$ & 0,08 & 0,38 & 0,36 & 0,44 & 0,65 & 0,29 & 0,13 & $-0,08$ & 0,06 & $-0,12$ & $-0,02$ & 0,58 \\
\hline Zamora & 0,07 & 0,08 & 0,52 & 0,42 & 0,55 & 0,69 & 0,48 & 0,33 & 0,04 & 0,22 & 0,00 & 0,15 & 0,76 \\
\hline
\end{tabular}

Blanco: correlación no significativa. Gris claro: tendencia significativa con un nivel de confianza del 95

$\%$. Gris oscuro: tendencia significativa con un nivel de confianza del $99 \%$

El análisis que muestran los CUADROS 8 y 9 respectivamente sugiere que las temperaturas medias de las máximas han tenido un mayor peso en el incremento de las temperaturas medias que las temperaturas medias de las mínimas. Tanto para el conjunto del período de estudio (1961-2006), como para el período 1973-2006, los coeficientes de correlación de Spearman anuales son positivos y estadísticamente significativos en el caso de las temperaturas medias de las máximas, destacando de nuevo marzo y junio en el análisis intraanual. En el caso de las temperaturas medias de las mínimas en 4 observatorios no se detecta ningún tipo de tendencia en las medias anuales para el conjunto del período, aunque si se analiza la subserie 1973-2006 el significado estadístico del análisis aumenta de manera apreciable, ya que sólo en el caso de Ávila no se alcanza un nivel de confianza del $95 \%$. El análisis intraanual de las temperaturas medias de las mínimas apunta a los meses de junio, mayo, marzo y octubre como los 
más sensibles a los cambios. Promediando los valores correspondientes a los 9 observatorios meteorológicos, entre 1973 y 2006 las temperaturas medias de las máximas subieron a un ritmo de $0,056^{\circ} \mathrm{C} / \mathrm{año}$, mientras que en el caso de las medias de las mínimas el ratio de subida fue menor $\left(0,035^{\circ} \mathrm{C} / \mathrm{año}\right)$.

Cuadro 9. Coeficientes de correlación de Spearman correspondientes a las series de temperaturas medias mínimas mensuales y anuales. Cuadro a: resultados correspondientes a la totalidad del periodo de estudio (1961-2006). Cuadro b: resultados correspondientes al periodo 1973-2006.

\begin{tabular}{lcc|c|c|c|c|c|c|c|c|c|c}
\hline \multicolumn{10}{c}{ Cuadro a: Temperatura medias de las mínimas $(1961-2006)$} \\
\hline
\end{tabular}

Blanco: correlación no significativa. Gris claro: tendencia significativa con un nivel de confianza del 95 $\%$. Gris oscuro: tendencia significativa con un nivel de confianza del $99 \%$

\section{Comportamiento de las precipitaciones}

A pesar del signo negativo de la mayoría de los coeficientes de Spearman (CUADRO 10), el análisis estadístico de la evolución temporal de las precipitaciones no muestra ninguna tendencia significativa, salvo en el caso de Ávila, en donde, en contra de la tendencia predominante, las precipitaciones totales anuales han registrado un incremento del $30 \%$ a lo largo del período de estudio, con un intervalo de confianza del $99 \%$. En el resto de los observatorios las variaciones han oscilado entre un incremento del $3 \%$ en Segovia y un decrecimiento del $17 \%$ en Valladolid. Aunque en algunos observatorios como Burgos, Palencia, Segovia, Soria, Valladolid y Zamora (FIGURA 3) se aprecia una tendencia decreciente, entre 1961 y 1991, y ascendente, entre 1992 y 2006, éstas tampoco son significativas desde un punto de vista estadístico.

El análisis de la evolución temporal de la precipitación en los distintos meses muestra que en 6 de las 9 series analizadas, las lluvias están decreciendo de 
forma estadísticamente significativa en el mes de febrero. Por término medio, las precipitaciones correspondientes al mes de febrero nunca representan más del $9 \%$ del total anual frente a los meses más lluviosos (noviembre y octubre) con más del $11 \%$. En agosto se registra una tendencia creciente estadísticamente significativa en 5 de las 9 series, aunque la incidencia de esta circunstancia es mínima debido a la escasa cuantía e irregularidad de las precipitaciones en este mes.

Cuadro 10. Coeficientes de correlación de Spearman correspondientes a las series de precipitaciones mensuales y anuales.

\begin{tabular}{|c|c|c|c|c|c|c|c|c|c|c|c|c|c|}
\hline \multicolumn{14}{|c|}{ Precipitaciones (1961-2006) } \\
\hline & Ene & Feb & Mar & $A b r$ & May & Jun & Jul & Ago & Sep & Oct & Nov & Dic & Año \\
\hline Ávila & 0,00 & 0,01 & 0,05 & 0,02 & 0,45 & $-0,10$ & $-0,04$ & 0,26 & $-0,04$ & 0,36 & 0,13 & 0,29 & 0,44 \\
\hline Burgos & $-0,20$ & $-0,34$ & $-0,23$ & $-0,01$ & $-0,12$ & $-0,09$ & $-0,05$ & 0,06 & 0,00 & 0,32 & 0,01 & 0,14 & $-0,16$ \\
\hline León & $-0,17$ & $-0,42$ & $-0,01$ & $-0,07$ & 0,12 & $-0,22$ & 0,04 & 0,31 & 0,07 & 0,15 & $-0,09$ & 0,08 & $-0,11$ \\
\hline Palencia & $-0,07$ & $-0,42$ & $-0,20$ & 0,04 & 0,07 & $-0,32$ & $-0,16$ & 0,49 & 0,23 & 0,35 & 0,07 & 0,03 & $-0,06$ \\
\hline Salamanca & $-0,16$ & $-0,29$ & $-0,25$ & 0,00 & 0,18 & $-0,20$ & $-0,05$ & 0,20 & $-0,06$ & 0,26 & $-0,13$ & 0,05 & $-0,12$ \\
\hline Segovia & $-0,12$ & $-0,13$ & $-0,24$ & 0,00 & 0,26 & $-0,04$ & $-0,02$ & 0,37 & $-0,19$ & 0,34 & $-0,09$ & 0,12 & 0,09 \\
\hline Soria & $-0,18$ & $-0,26$ & $-0,15$ & $-0,07$ & 0,25 & $-0,21$ & 0,04 & 0,19 & $-0,01$ & 0,33 & $-0,11$ & $-0,07$ & $-0,08$ \\
\hline Valladolid & $-0,13$ & $-0,32$ & $-0,21$ & $-0,07$ & 0,01 & $-0,30$ & $-0,12$ & 0,47 & $-0,08$ & 0,30 & $-0,08$ & 0,02 & $-0,22$ \\
\hline Zamora & $-0,09$ & $-0,32$ & $-0,16$ & 0,05 & 0,09 & $-0,35$ & 0,00 & 0,32 & 0,00 & 0,29 & $-0,03$ & 0,08 & $-0,07$ \\
\hline
\end{tabular}

Blanco: correlación no significativa. Gris claro: tendencia significativa con un nivel de confianza del 95

$\%$. Gris oscuro: tendencia significativa con un nivel de confianza del $99 \%$

\section{Discusión y conclusiones}

Los resultados obtenidos en el presente trabajo coinciden de forma general con las tendencias observadas globalmente respecto a las principales variables climáticas (IPCC, 2007). No obstante, bien matizan o bien corroboran algunas conclusiones respecto a la estacionalidad del aumento de las temperaturas, el peso específico de las temperaturas medias máximas y mínimas y la tendencia de las precipitaciones.

A nivel global y regional una diversidad de estudios que analizan la evolución de las temperaturas con series de datos largas (más de 100 años) subrayan la inflexión de las anomalías térmicas a comienzos de los 70 del siglo pasado, con una tendencia creciente a partir de 1973 (p.e. GALÁN ET AL., 2001; MILLY ET AL., 2005; DE CASTRO ET AL., 2005; MORALES ET AL., 2005; JONES ET AL., 2006 O BRUNET ET AL., 2007). En el caso del territorio español peninsular, BRUNET ET AL. (2007) han analizado una serie homogénea a partir de los datos de 22 observatorios para el período 1850-2005 concluyendo que durante los últimos 100 años se han registrado dos períodos de incremento térmico significativo (1901-1949 y 1973-2005) interrumpidos por un período de descenso térmico (1950-1972), que, con algunos matices, coincide con la tendencia observada a escala global (JONES ET AL.; 2006). 
Aunque los ratios anuales de calentamiento están determinados por el ámbito espacial del análisis y el número de años utilizados en el ajuste lineal, el valor medio obtenido en nuestro estudio $\left(0,043{ }^{\circ} \mathrm{C} /\right.$ año para el período $\left.1973-2006\right)$ está por encima del general estimado por JONES ET AL. (2006), algo por debajo del apuntado por DE CASTRO ET AL. (2005) para el territorio español $(0,058$ ${ }^{\circ} \mathrm{C} / \mathrm{año}$ ) y bastante próximo al publicado por MORALES ET AL. (2005) para la Comunidad de Castilla y León $\left(0,049{ }^{\circ} \mathrm{C} / \mathrm{año}\right)$.

Aunque la mayor parte de los análisis previos estudian la tendencia intraanual de las temperaturas por estaciones y no por meses, hemos creído conveniente analizar las temperaturas mes por mes debido a las diferencias existentes entre meses comprendidos en una misma estación, apreciable sobre todo en la evolución temporal de las temperaturas medias de las mínimas (CUADRO 9). De forma general, y sobre todo a partir de 1973, el incremento de las temperaturas ha sido evidente en las estaciones primaveral y estival, destacando los meses de marzo y junio. Esta percepción coincide con el análisis de BRUNET ET AL. (2007) para el conjunto del territorio español, quienes, concretamente en el subperíodo 1973-2005, subrayan un aumento de las temperaturas medias estadísticamente significativo en primavera y verano, mientras que en otoño e invierno la tendencia no alcanza un intervalo de confianza superior al $95 \%$. A escala regional (Castilla y León), resultados parecidos fueron sugeridos por MORALES ET AL. (2005) para un período de 25 años (1972-1996).

Si bien en el conjunto del territorio español peninsular STAUDT (2004) y BRUNET ET AL. (2007) coinciden en cuantificar un incremento paralelo de las temperaturas medias de máximas y mínimas en el período 1973-2005, en nuestro caso hemos detectado un comportamiento distinto en concordancia con el observado en otros espacios regionales como la meseta meridional (GALÁN ET AL., 2001). La diferencia constatada entre el ritmo anual de incremento de las temperaturas medias de las máximas $\left(0,056^{\circ} \mathrm{C}\right)$ y de las mínimas $\left(0,035^{\circ} \mathrm{C}\right)$ es mayor que la detectada por MORALES ET AL. (2005) a escala regional, lo cual probablemente se deba a que en la última década, excepto en los casos de Ávila y Burgos (FIGURA 2), la tendencia de las mínimas haya sido nula o incluso decreciente, mientras que en el caso de las máximas haya sido predominantemente creciente.

Nuestros resultados no corroboran las conclusiones de algunos trabajos que afirman, de forma general en el territorio español, que un incremento notable de las temperaturas mínimas ha sido el responsable del incremento observado en las temperaturas medias, mientras que las temperaturas máximas no han mostrado una tendencia temporal significativa (ESTEBAN-PARRA ET AL., 1995). Tampoco confirman las conclusiones de OÑATE Y POU (1995), quienes observan una tendencia negativa de las temperaturas medias máximas en los meses 
primaverales y estivales en los observatorios interiores de la Península Ibérica. Los trabajos de Rodríguez-PuEBLA ET AL. (2002), STAUDT (2004) y DE CASTRO ET AL. (2005), en la línea del anterior, apuntan que el calentamiento ha afectado sobre todo a los meses invernales, mientras que en nuestro análisis no hemos observado ninguna tendencia significativa en estos meses, salvo en el caso de diciembre y, paradójicamente, considerando la totalidad del período y no los últimos 34 años. Es decir, cuando más evidente ha sido el calentamiento, menor ha sido la aportación de los registros invernales al mismo. Estas contradicciones demuestran el interés de generar análisis regionales que maticen o corroboren las conclusiones de estudios de ámbito general.

El trabajo de SERRANO ET AL. (1999), analizando datos correspondientes a 40 observatorios durante el período 1921-1995, concluía que para el conjunto de la Península Ibérica no se observaba ninguna tendencia temporal significativa en el caso de la precipitación anual y sólo en la mitad de los casos se detectaba un descenso de la precipitación apreciable en el mes de marzo. Trabajos posteriores han confirmado estas apreciaciones: la síntesis de DE CASTRO ET AL. (2005) ilustra, mediante numerosos casos, la ausencia de tendencias en las precipitaciones peninsulares, a pesar de la percepción general de que las lluvias han decrecido en el conjunto de la región mediterránea (IPCC, 2001), y PAREDES ET AL. (2006) insisten en la reducción significativa de las precipitaciones del mes de marzo, aunque en ninguno de los observatorios estudiados en nuestro trabajado se haya observado este patrón.

Nuestros resultados son similares a los publicados en diversos trabajos que han analizado la tendencia temporal de las precipitaciones en distintos espacios regionales. En la Comunidad Valenciana, HIDALGO ET AL. (2001) encuentran una gran heterogeneidad en el comportamiento de las 97 series analizadas, aunque destaca el predominio de casos en los que se no se detectó ningún tipo de tendencia estadísticamente significativa. Tampoco encuentran tendencia alguna LÁZARO ET AL. (2001) en su análisis pluviométrico del extremo sureste de la Península. SALADIE ET AL. (2002) apuntan en el sector suroriental de la depresión del Ebro un descenso de la precipitación anual del 12,3 \%, siendo la primavera la estación más sensible. No obstante, al igual que en nuestro caso, estas tendencias temporales tampoco han resultado estadísticamente significativas. Finalmente el estudio de LABAJO Y PIORNO (2001) que analiza una serie pluviométrica homogeneizada, a partir de 44 estaciones pluviométricas en el período 1931-1996, tampoco detecta tendencia temporal alguna en las series estacionales y anuales en el caso de Castilla y León.

Numerosos investigadores están haciendo un notable esfuerzo por encontrar patrones en el comportamiento del sistema océano-atmósfera para explicar estos cambios. De este modo, RODRÍGUEZ-PUEBLA ET AL. (2002) destacan la impor- 
tancia del índice NAO (North Atlantic Oscillation) y el denominado Iberian Oscillation Index para entender la evolución de las temperaturas en los meses más fríos del año, mientras que la temperatura superficial del mar tendría un papel relevante en la dinámica de las temperaturas estivales. En el caso de las precipitaciones, trabajos como el de MARTÍN-VIDE Y FERNÁNDEZ-BELMONTE (2001) encuentran una buena correlación entre el índice NAO y las precipitaciones mensuales en la España peninsular, especialmente en la región centro y suroccidental entre los meses de octubre y marzo.

Las conclusiones de nuestro análisis se podrían resumir muy brevemente en que i) las temperaturas medias mensuales y anuales han subido de forma clara en todas las capitales de Castilla y León a partir de de 1973; ii) los meses de marzo y junio han sido los que han registrado un mayor incremento en sus valores térmicos; iii) las temperaturas medias de las máximas han subido más que las medias de las mínimas y iv) no se observa ninguna tendencia estadísticamente significativa en el caso de las precipitaciones, aunque de forma general su evolución temporal haya sido decreciente.

Aunque no es nuestro objetivo valorar las posibles consecuencias de estos cambios, en el contexto de nuestra comunidad autónoma, las tendencias observadas en temperaturas y precipitaciones pueden derivar en las siguientes implicaciones:

El aumento de las temperaturas en primavera está adelantando la ocurrencia de numerosos procesos de orden fenológico a un ritmo de 2,3 días por década (WALTER ET AL., 2002; PARMESAN Y YOHE, 2003), siendo uno de los más evidentes los cambios en la fecha de foliación de muchas especies vegetales (MENZEL ET AL., 2006). En el caso de Castilla y León, ello implicaría el adelanto del brote de las hojas en los bosques marcescentes, predominantes en la región, de Quercus pyrenaica en algo más de 11 días en las últimas 5 décadas, con un consumo extra de agua que en cuencas experimentales se ha estimado en $38 \mathrm{~mm}$. Este consumo de agua implica i) que la reserva hídrica del suelo decrezca rápidamente, alcanzando en un intervalo de pocos días una situación de apreciable déficit (MARTÍNEZ-FERNÁNDEZ ET AL., 2005) y ii) que en el balance hídrico la interceptación e infiltración prevalezcan sobre la escorrentía superficial, con una evidente disminución de los recursos hídricos superficiales.

Otra consecuencia de este incremento de las temperaturas en primavera y verano es el estrés que sufren muchos cultivos de secano. El caso más evidente es el de los cereales y el asuramiento del trigo en mayo y junio, debido a lo cual el secano comienza a ser tratado como cultivo de regadío, con un notable incremento de costes económicos (costes de energía) y ambientales (reducción de las reservas de agua). 
Finalmente, aunque el comportamiento de las precipitaciones no tenga una trayectoria clara como en el caso de las temperaturas, la disminución significativa de las lluvias en febrero, combinada con el aumento de las temperaturas en los meses primaverales (principalmente marzo), está repercutiendo en un cambio apreciable en el régimen de los ríos, con un adelantamiento del comienzo del estiaje y una menor disponibilidad de recursos hídricos superficiales en el conjunto de las cuencas (CEBALLOS ET AL, 2008).

\section{Agradecimientos}

Esta investigación ha sido posible gracias al apoyo financiero de la Junta de Castilla y León (Proyecto SA099A06). También queremos expresar nuestro sincero agradecimiento a dos revisores anónimos cuyos comentarios y sugerencias nos han permitido mejorar la versión original de nuestro trabajo.

\section{Referencias bibliográficas}

Aguilar, E.; LóPez J.M.; Brunet, M.; SAladiÉ, O.; Sigró, J. y LóPez, D. (1999): «Control de calidad y proceso de homogenización de series térmicas catalanas». En RASO, J.M. y MARTÍN VIDE, J. (Eds): La climatología española en los umbrales del siglo XXI. Publicaciones de la Asociación Española de Climatología. AEC, serie A, 1, pp. 15-23.

Beguería, S.; LóPEZ-MORENo, J. I.; LORENTE, A.; SEEgER, M. y GARCíA-RUiZ, J. M. (2003): "Assessing the effect of climate oscillations and land-use changes on streamflow in the Central Spanish Pyrenees». Ambio, 32(4), pp. 283-286.

Brunet, M.; Jones, P.D.; Sigró, J.; SAladié, O.; Aguilar, E. ; Moberg, A. DellaMARTA, P.M.; LISTER, D.; WALTER, A. Y LÓPEZ, D. (2007): «Temporal and spatial temperature variability and change over Spain during 1850-2005». Journal of Geophysical Research, 112, D12117.

Ceballos, A.; Morán Tejeda, E.; Luengo Ugidos, M. A. y Llorente Pinto, J.M. (2008): «Water resources and environmental changes in a Mediterranean environment: South West Sector of the Duero river basin (Spain)». Journal of Hydrology, 351, pp. 126-138.

De CASTRO, M.; MARTín VIDE, J. Y AlONSO, S. (2005): «El clima de España: pasado, presente y escenarios de clima para el siglo XXI». En MORENO RodRíGUEZ, J.M. (Coord.): Evaluación preliminar de los impactos en España por efecto del cambio climático. Ministerio de Medio Ambiente y Universidad de Castilla-La Mancha, pp. $1-64$.

ESTEBAN-PARRA, M.J.; RODRIGO, F.S. Y CASTRO-DÍEZ, Y. (1995): «Temperature trends and change points in the Northern Spanish plateau during the last 100 years». International Journal of Climatology, 15, pp. 1031-1042.

GALÁN, E.; CAÑADA, R.; FERNÁNDEZ, F. Y CERVERA, B. (2001): «Annual temperature evolution in the southern plateau of Spain from the construction of regional climatic time series». En BRUNET, M. \& LóPEZ BonILLO, D. (Eds.): Detecting and Modelling Regional Climate Change. Springer. pp. 163-173. 
GonZÁlez HidAlgo, J.C.; DE Luis, M.; RAVEnTÓS, J. Y SÁnCHEZ, J. R. (2001): Spatial distribution of seasonal rainfall trends in a western Mediterranean area. International Journal of Climatology, 22, pp. 1593-1615.

IPCC (2001): Climatic Change 2001: The Scientific Bases. Contribution of Working Group I to the Third Assessment Report of the Intergovernmental Panel on Climate Change. Houghton, J.T.; Ding, Y.; Griggs, D.J.; Noguer, M.; VAn DER Linden, P.J.; DAI, X.; MASKell, K. y JOHNSON, C.A. (Eds). Cambridge University Press, United Kingdom.

IPCC (2007): Climatic Change 2007: The Physical Science Basis. Summary for Policymakers. Contribution of Working Group I to the Fourth Assessment Report to the Intergovernmental Panel on Climate Change. http://www.ipcc.ch

Jones, P.D., D.E. PARKER, T.J. OSBORN, Y K.R. BRIFFA (2006) «Global and hemispheric temperature anomalies--land and marine instrumental records». In Trends: A Compendium of Data on Global Change. Carbon Dioxide Information Analysis Center, Oak Ridge National Laboratory, U.S. Department of Energy, Oak Ridge, Tenn., U.S.A. http://cdiac.ornl.gov/trends/temp/jonescru/jones.html

KLEIN TANK, A.M.G. ET AL. (2002): «Daily dataset of 20th-century surface air temperature and precipitation series for the European Climate Assessment». International Journal of Climatology, 22, pp. 1441-1453.

LABAJO, J.L. Y PIORNO, A. (2001): «Regionalisation of precipitation in Castilla and Leon (Spain). Analysis of its temporal behaviour». En BRUNET, M. \& LóPEZ BonILlo, D. (Eds.): Detecting and Modelling Regional Climate Change. Springer, pp. 163-173.

LÁZARO, R.; Rodrigo, F.S.; Gutiérrez, L.; Domingo, F. Y PUIGdeFÁbregas, J. (2001). «Analysis of a 30-year rainfall record (1967-1997) in semi-arid SE Spain for implications on vegetation». Journal of Arid Environments, 48, pp. 373-395.

MARTÍN VIDE, J. Y FERNÁNDEZ BELMONTE, J. (2001): «El índice NAO y la precipitación mensual en la España peninsular». Investigaciones Geográficas, 26, pp. 41-58.

MARTínez Fernández, J.; Ceballos, A.; CASAdo, S.; Morán, C. Y HERnÁndez, V. (2005): «Runoff and soil moisture in a small forested basin in the Sistema Central Ranges (Spain)», in: BATALlA, R., GARCÍA, C. (Eds.), Geomorphological Processes and Human Impacts in River Basins. IAHS Publ. 299, pp. 31-36.

MENZEL, A. ET AL. (2006): «European phenological response to climate change matches the warming pattern». Global Change Biology, 12(10), pp. 1969-1976.

Milly, P.C.D.; DuNNE, K.A. Y VECCHIA, A.V. (2005): "Global pattern of trends in streamflow and water availability in a changing climate». Nature, 438/17: 347-350.

Morales, C.; OrTEGA, M.T.; LABAJO, J.L. Y PiORNO, A. (2005): «Recent trends and temporal behavior of thermal variables in the region of Castilla-León (Spain)». Atmósfera, 18(2), pp. 71-90.

MORENO RODRÍGUEZ, J.M. (Coord.) (2005): Evaluación preliminar de los impactos en España por efecto del cambio climático. Ministerio de Medio Ambiente y Universidad de Castilla-La Mancha.

OÑATE, J.J. Y POU, A. (1995): «Temperature variations in Spain since 1901: a preliminary analysis». International Journal of Climatology, 16, pp. 805-815. 
Paredes, D.; Trigo, R.M., García-Herrera, R. Y Franco Trigo, I. (2006): «Understanding Precipitation Changes in Iberia in Early Spring: Weather Typing and Storm-Tracking Approaches». Journal of Hydrometeorology, 7(1): 101-113.

PARMESAN, C. Y YOHE, G. (2003): «A globally coherent fingerprint of climate change impacts across natural systems». Nature, 421, pp. 37-42.

Rodríguez-Puebla, C.; García-CASAdo, L. A.; FríAs, M. D.; SÁenZ, J. Y ZUBILlAGA, J. (2002): «Trend and interannual variations in air temperatura over Iberian Peninsula, P 1.9». 13th Symposium on Global Change and Climate Variations. American Meteorological Society, Orlando, Florida (USA).

SALADIÉ, O.; BRUNET, M.; AgUILAR, E.; SigRÓ, J. Y LÓPEZ, D. (2002): «Evolución de la precipitación en el sector suroriental de la depresión del Ebro durante la segunda mitad del siglo XX». En J.A. GuiJarro, M. Grimalt, M. LAita y S. AlONSO (Eds.): El agua y el clima. pp. 335-346. Publicaciones de la Asociación Española de Climatología, (AEC), Serie A, ${ }^{\circ} 3$.

SCIAN, B. Y DONNARI, M. (1997): «Retrospective analysis of the palmer Drought Severity Index in the semi-arid pampas region, Argentina». International Journal of Climatology, 17, pp. 313-322.

SERRANO, A.; MATEOS, L. Y GARCÍA, J.A. (1999): «Trend analysis of monthly precipitation over the Iberian Peninsula for the period 1921-1995». Physics and Chemistry Earth, 24(1-2), pp. 85-90.

Shorthouse, C. Y ARnell, N. (1999): «The effects of climate variability on spatial characteristics of European river flows». Physics and Chemistry Earth, 24(1-2), pp. 7-13.

SNEYERS, R. (1992): «Use and misuse of statistical methods for detection of climatic change». In: Climate Change Detection Project, Report on the Informal Planning Meeting on Statistical Procedures for Climate Change Detection. WCDMP, (20); J76-J81.

STAudt, M. (2004): Detección de cambios térmicos en la Península Ibérica con datos homogéneos regionales. Tesis Doctoral Inédita. Departamento de Física Aplicada, Universidad de Granada. 559 pp.

ToMÁs, C.; SÁNCHEZ, M.C. Y DE PABLO, F. (1998): «La precipitación sobre Zamora, 1920-1997. Variaciones mensuales, estacionales y anuales». Anuario del Instituto de Estudios Zamoranos “Florián de Ocampo”. pp. 327-339.

Walter, G-R.; Post, E.; Convey, P.; Menzel, A.; Parmesan, C.; Beebee, T.; Fromentin, J-M.; HoEGH-GuldBerG, O. Y BAIRLEIN, F. (2002): «Ecological responses to recent climate change». Nature, 416, pp. 389-395.

Yue, S.; Pilon, P. Y CAVAdias, G. (2002): «Power of the Mann-Kendall and Spearman's rho tests for detecting monotonic trends in hydrological series». Journal of Hydrology, 259, pp. 254-271. 
Figura 1. Localización de las capitales de provincia en Castilla y León

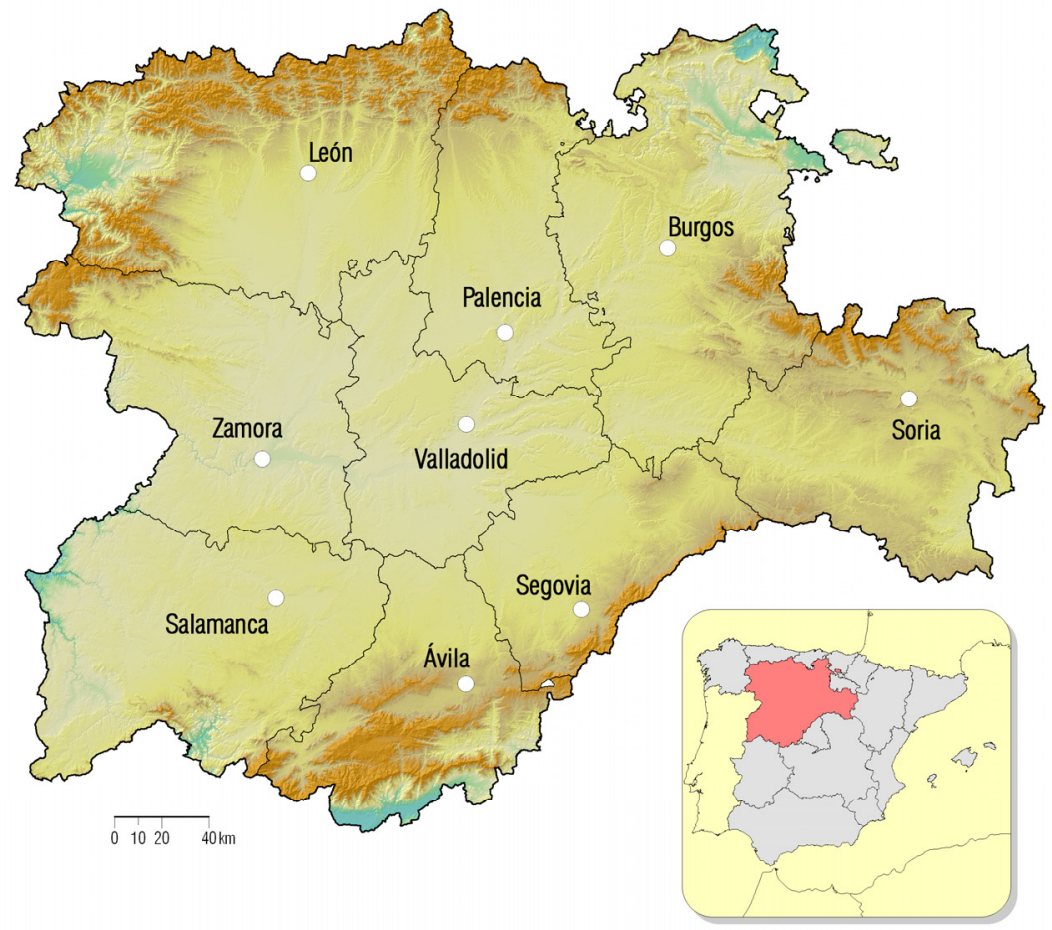


Figura2. Anomalias térmicas anuales respecto al periodo de referencia 1961-2006 en las nueve capitales provinciales de Castilla y León (medias móviles de 5 años).
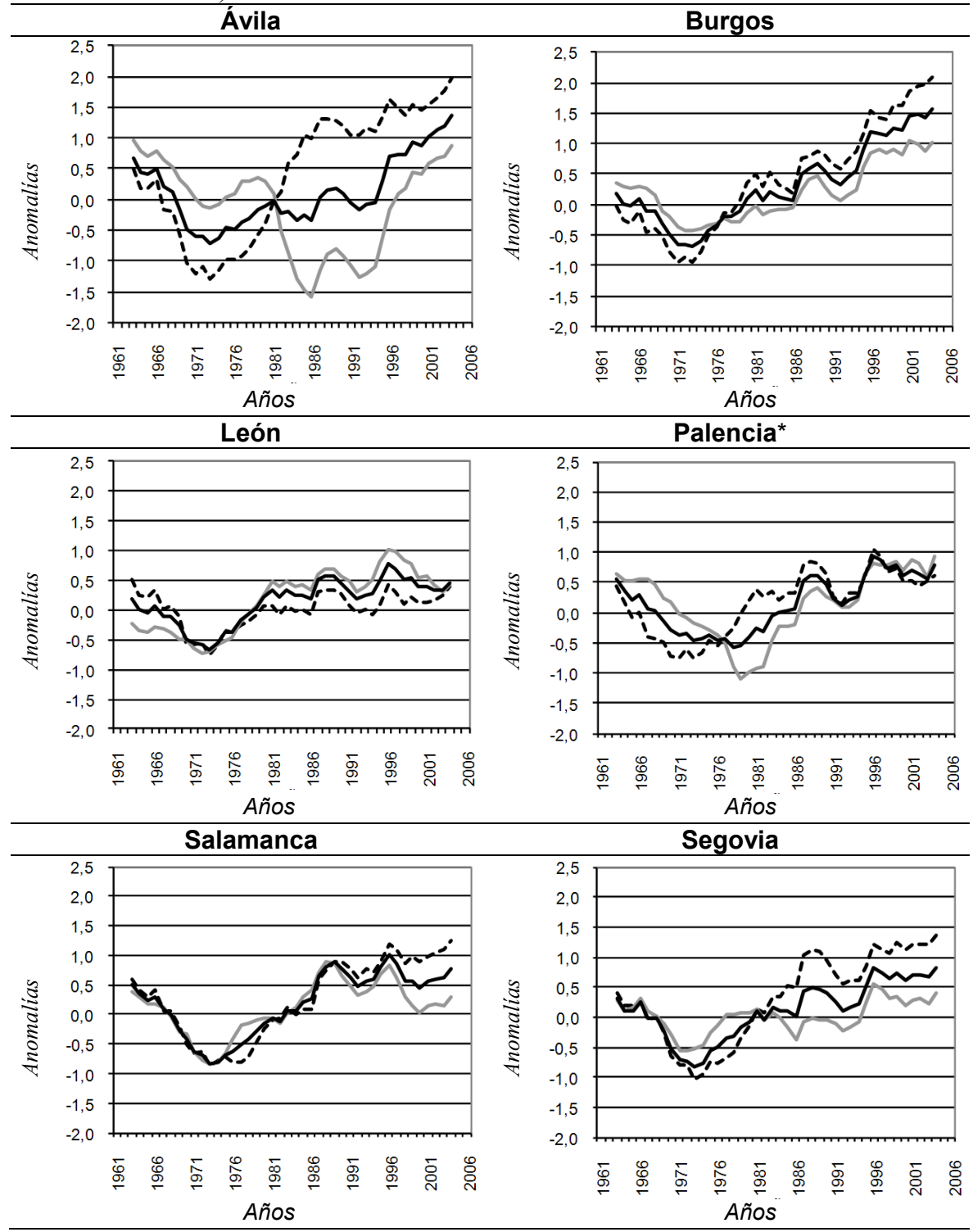
Figura2. (cont.) Anomalias térmicas anuales respecto al periodo de referencia 19612006 en las nueve capitales provinciales de Castilla y León (medias móviles de 5 años).
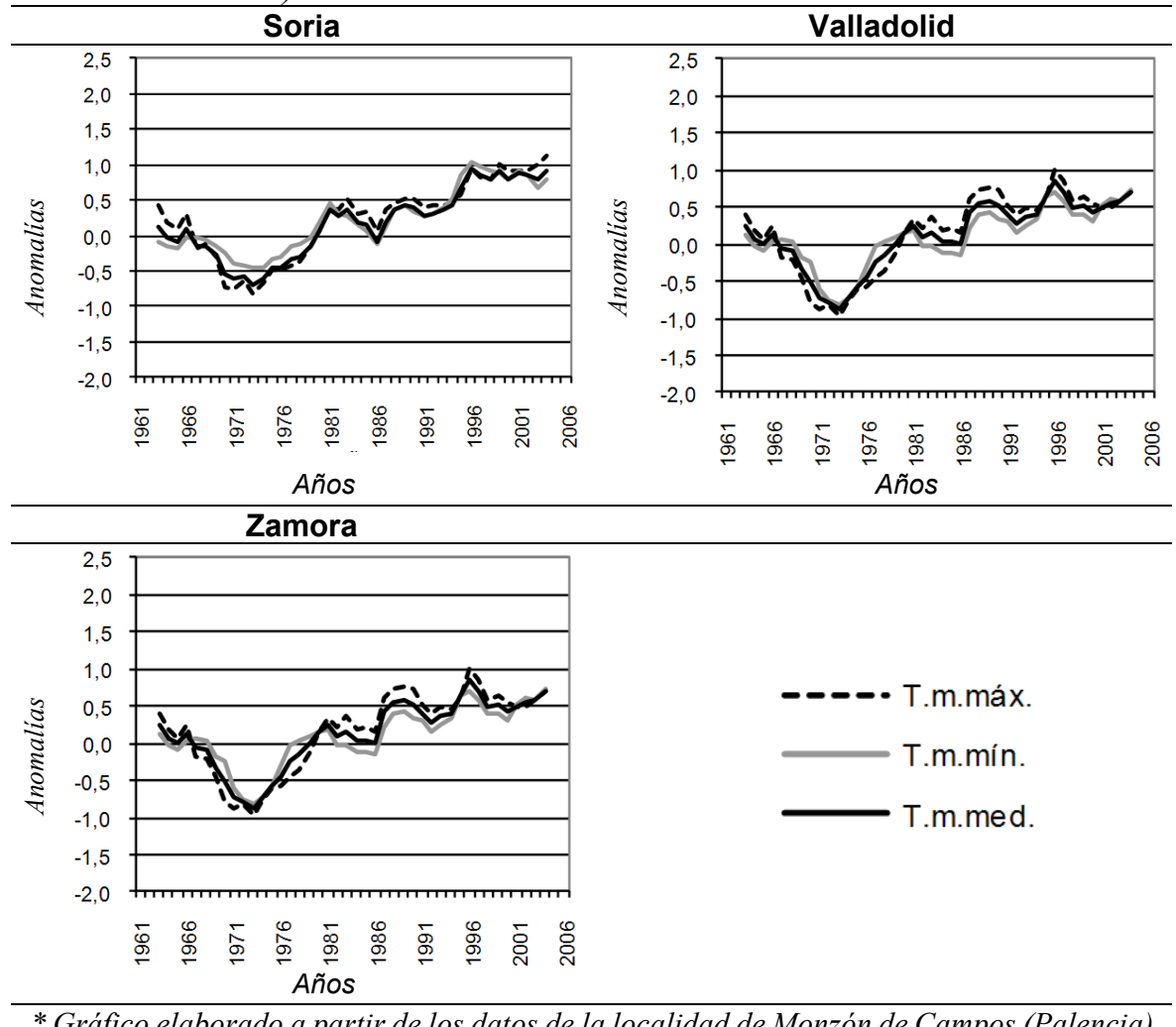

* Gráfico elaborado a partir de los datos de la localidad de Monzón de Campos (Palencia) 
Figura 3. Evolución interanual de las precipitaciones en las nueve capitales provinciales de Castilla y León (1961-2006). Línea gris: media móvil de 9 años. Líneas negras: precipitación media $\pm S D$
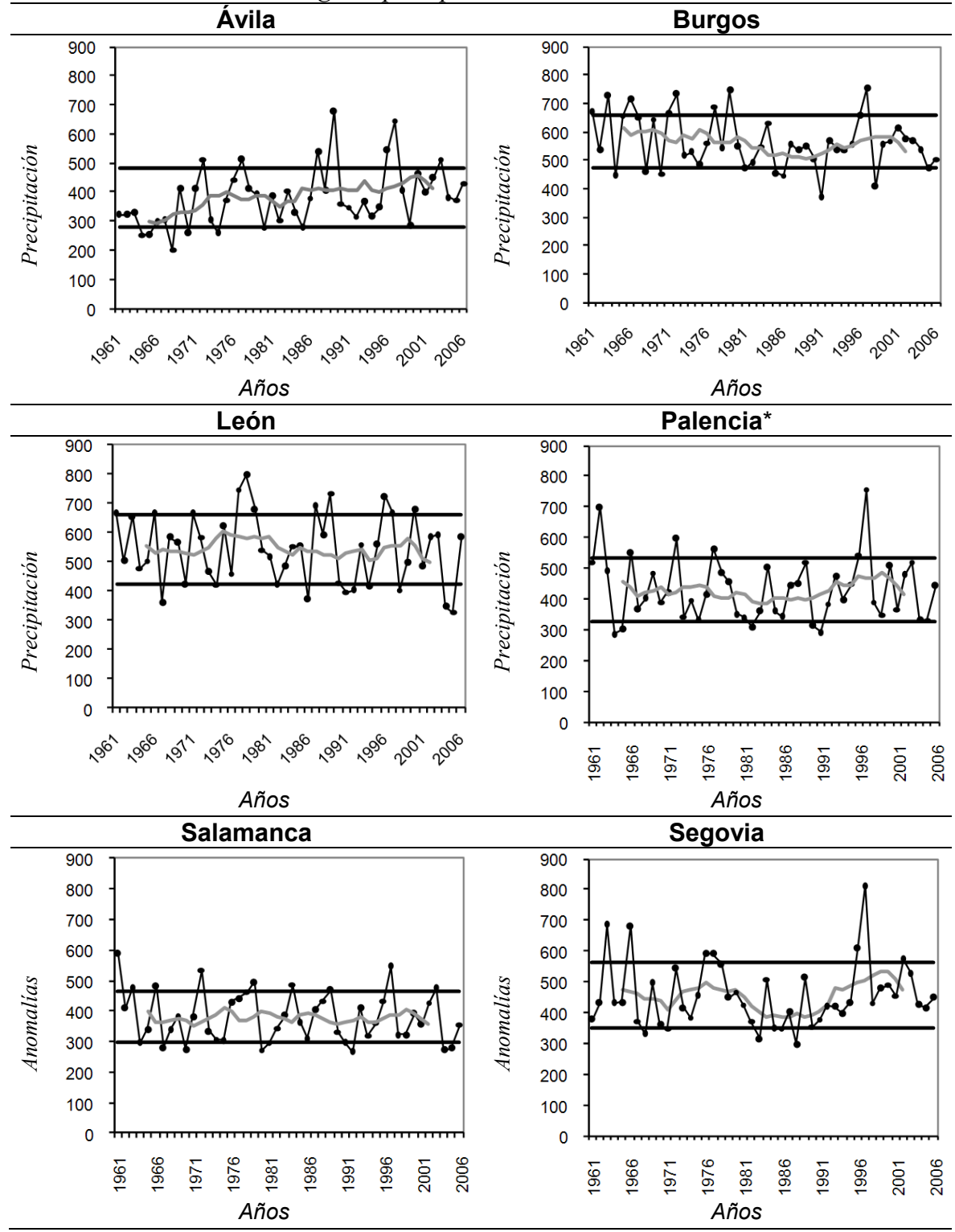
Figura 3. (cont.) Evolución interanual de las precipitaciones en las nueve capitales provinciales de Castilla y León (1961-2006). Línea gris: media móvil de 9 años. Lineas negras: precipitación media $\pm S D$

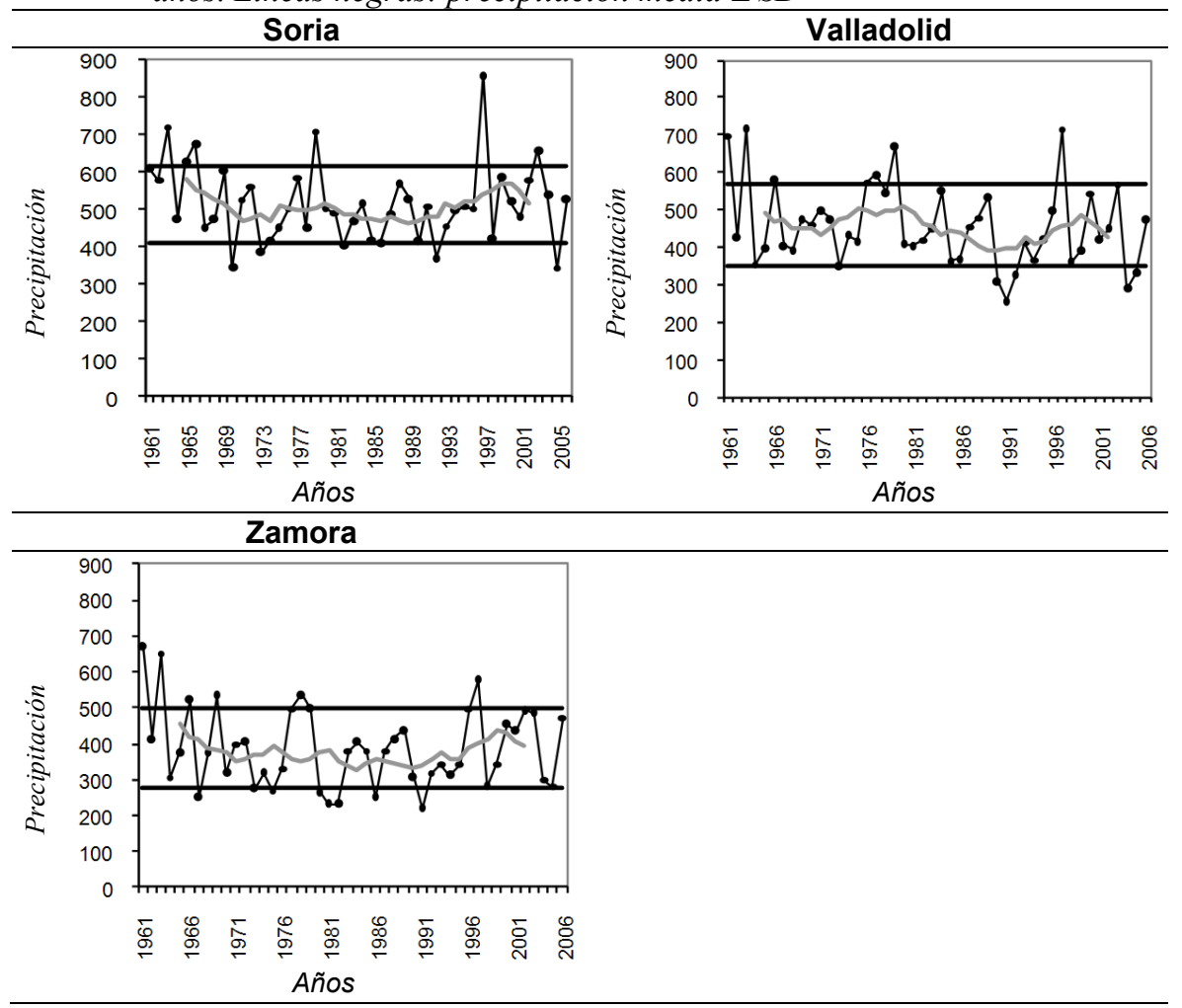

